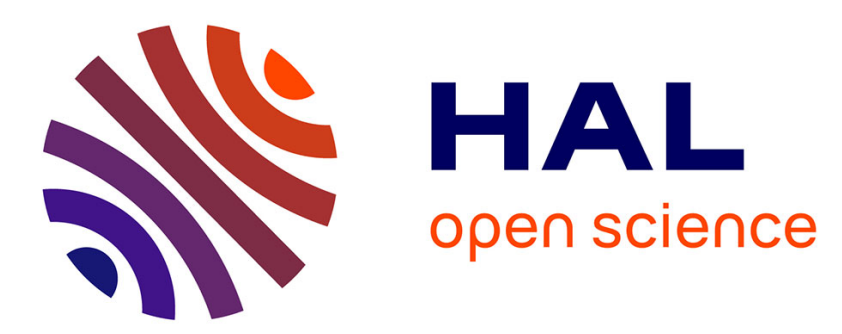

\title{
Efficient Pruning LMI Conditions for Branch-and-Prune Rank and Chirality-Constrained Estimation of the Dual Absolute Quadric
}

\author{
Adlane Habed, Danda Pani Paudel, Cédric Demonceaux, David Fofi
}

\section{- To cite this version:}

Adlane Habed, Danda Pani Paudel, Cédric Demonceaux, David Fofi. Efficient Pruning LMI Conditions for Branch-and-Prune Rank and Chirality-Constrained Estimation of the Dual Absolute Quadric. IEEE Conference on Computer Vision and Pattern Recognition, Jun 2014, United States. pp.1-6. hal-01017687

\section{HAL Id: hal-01017687 \\ https://hal.science/hal-01017687}

Submitted on 2 Jul 2014

HAL is a multi-disciplinary open access archive for the deposit and dissemination of scientific research documents, whether they are published or not. The documents may come from teaching and research institutions in France or abroad, or from public or private research centers.
L'archive ouverte pluridisciplinaire HAL, est destinée au dépôt et à la diffusion de documents scientifiques de niveau recherche, publiés ou non, émanant des établissements d'enseignement et de recherche français ou étrangers, des laboratoires publics ou privés. 


\section{Efficient Pruning LMI Conditions for Branch-and-Prune Rank and Chirality-Constrained Estimation of the Dual Absolute Quadric}

\author{
Adlane Habed \\ ICube laboratory/CNRS \\ University of Strasbourg, France \\ habedeunistra.fr
}

\author{
Danda Pani Paudel Cédric Demonceaux David Fofi \\ Le2i laboratory/CNRS \\ University of Bourgogne, France \\ \{danda-pani.paudel, cedric.demonceaux, david.fofi\}@u-bourgogne.fr
}

\begin{abstract}
We present a new globally optimal algorithm for selfcalibrating a moving camera with constant parameters. Our method aims at estimating the Dual Absolute Quadric (DAQ) under the rank-3 and, optionally, camera centers chirality constraints. We employ the Branch-and-Prune paradigm and explore the space of only 5 parameters. Pruning in our method relies on solving Linear Matrix Inequality (LMI) feasibility and Generalized Eigenvalue $(G E V)$ problems that solely depend upon the entries of the $D A Q$. These LMI and GEV problems are used to rule out branches in the search tree in which a quadric not satisfying the rank and chirality conditions on camera centers is guaranteed not to exist. The chirality LMI conditions are obtained by relying on the mild assumption that the camera undergoes a rotation of no more than $90^{\circ}$ between consecutive views. Furthermore, our method does not rely on calculating bounds on any particular cost function and hence can virtually optimize any objective while achieving global optimality in a very competitive running-time.
\end{abstract}

\section{Introduction}

Self-calibration (retrieving camera's intrinsic parameters from image correspondences) of a moving camera is a nonlinear and challenging problem that has been the subject of extensive research and exciting developments during the past two decades. Most approaches rely on the ubiquitous nature of the so-called Absolute Conic (AC): a special conic lying on the plane at infinity. Early methods [11] exploited the epipolar relationship between the duals of the images of the AC (DIACs) which encode the parameters of the imaging cameras. Relying on a set of camera matrices that are consistent with the same projective scene structure allows to cope with the artificial degeneracies arising when using epipolar geometry. Given such cameras/scene set, chirality inequalities [9] have been used to define bounds on the location of the plane at infinity allowing for an exhaustive search alternating location hypotheses and parameter estimation. A similar approach, iterating however on the space of intrinsic parameters, has also been proposed in [6]. When the camera parameters are constant though, stratified camera self-calibration can be employed to retrieve the plane at infinity by first solving the modulus constraints [13] before linearly retrieving the DIAC. Stratified methods contrast with the direct ones that aim at simultaneously locating the plane at infinity and the camera parameters. Direct methods rely on the omnipresence of a special virtual plane quadric, the Dual Absolute Quadric (DAQ) [15, 10], encoding the camera parameters and the plane at infinity.

Recent works on camera self-calibration [5, 3, 4, 1] have focused on devising deterministic globally convergent algorithms that only require bounds on some initial search space and that converge to a global minimum with an optimality certificate. Such methods are rather appealing as they sidestep the convergence issues that arise when using local optimization. Fusiello and Benedetti proposed in [5] a method based on Interval Analysis and employing the Branch-and-Bound (B\&B) algorithmic paradigm. Their method was developed to solve the Huang-Faugeras constraints on the essential matrix. However, with the degeneracies arising from the use of epipolar geometry, possibly increasing the number of branches, and the complexity of the considered constraints, the method has proved computationally quite expensive and applicable only to rather short image sequences. A B\&B-based approach, this time in conjunction with convex relaxations, was also used by Chandraker et al. [3] for stratified camera-self-calibration via the modulus and chirality constraints. The main drawback of this method lies in the fact that it employs two independent algorithms: one for locating the plane at infinity and another for estimating the DIAC. However, the modulus constraint is only a necessary condition on the location of the plane at infinity and likely to admit, in particular for short image sequences, more than one global minimizer. Hence, the global solution obtained from locating the plane at infinity, 
although guaranteed to be global, is not guaranteed to support a conic that identically project on all views. This may mainly occur because the search for the plane at infinity is tackled independently from finding the DIAC. Hence, a globally optimal algorithm for the self-calibration problem should simultaneously act on both locating the plane at infinity and the DIAC by typically solving the DAQ formulation of the problem. While the latter can be linearized under some camera parameters assumptions [14], it was shown in [7] that, by failing to enforce the DAQ's positiveness and rank-3 constraints, the linearized equations may yield artificial critical camera motions. In this regard, Gordjos et al. [7] proposed to relax the rank constraint and reintroducing it a posteriori while Bocquillon et al. [1] devised a globally optimal method for estimating the focal length of the camera. Another method, in which global optimality was sought, is the one presented by Chandraker et al. in [4] for solving the DAQ formulation of the self-calibration problem. This method not only carries the advantage of estimating the DAQ while enforcing its rank-degeneracy and positive semi-definiteness but also allows to enforce the chirality constraints as well during optimization. In particular, satisfying the chirality conditions on the camera centers insures, at each optimization iteration, that the candidate plane at infinity does not cut through the convex hull of cameras which may otherwise be detrimental to the viability of many cost functions used for camera self-calibration [12]. The constrained DAQ estimation in [4] is stated as a polynomial optimization problem subject to polynomial inequalities. The polynomial optimization method adopted therein initially requires to transform all constraints, should they be linear, into nonlinear polynomials (of a degree of at most 4) only to be linearized or re-linearized using lifting variables and constraints. The problem is handled by solving a hierarchy of nested Linear Matrix Inequality (LMI) relaxations of increasing order by gradually introducing lifting variables and constraints as to linearize monomials up to a given degree. Although the solutions of the LMI relaxations are guaranteed in theory to converge to the global optimum, the order of relaxation allowing for global convergence cannot be known in advance. Furthermore, with the rapidly increasing number of lifting variables and constraints, the problem may not even be numerically tractable when, or way before, the order of relaxation allowing for convergence is reached.

In this paper we present a new globally optimal method for the rank-constrained estimation of the DAQ possibly, under some mild camera motion conditions, in conjunction with chirality conditions on camera centers. Unlike [4], our method is applicable only in the case of a moving camera with constant intrinsic parameters. However, we show that, under such conditions and by exploiting the structure of the DAQ equations, a rather simple Branch-and-Prune (B\&P) algorithm involving LMIs with no more than 9 variables can be employed to achieve global optimality in a very competitive running-time in comparison with existing global methods. Given some bounds on the intrinsic parameters, our method proceeds by recursively branching and exploring the DIAC's parameters space (5 parameters) only to rule out branches in which a quadric projecting on the same DIAC on all views and exhibiting the desired rank - and possibly chirality properties - is guaranteed not to exist. The likely existence of such quadric within prescribed bounds is expressed via a set of LMI feasibility problems and LMI generalized eigenvalue (GEV) problems. All LMI and GEV problems are expressed in terms of at most 9 variables: the entries of the DAQ. This includes the rank-3 condition on the DAQ. We also show that the chirality of camera centers can be expressed by a set of LMIs in the entries of the DAQ under the assumption that the camera undergoes a rotation of no more than $90^{\circ}$ between consecutive views Note that this is a mild assumption implicitly verified when capturing images with the goal of carrying out point correspondences across consecutive pairs of images. Furthermore, our method can virtually be used with any objective since it does not rely on calculating bounds on any particular cost function. This is particularly important since the DAQ formulation introduces a different unknown scale with each view and using an appropriate, say normalized, cost function is highly recommended.

\section{Background and notation}

The scene is imaged by $n$ pinhole cameras represented, in some arbitrarily chosen projective frame, by a set of $3 \times$ 4 full row-rank camera matrices $\mathrm{P}_{\mathrm{i}}, i=1 \ldots n$. Planes, image and scene points are represented by homogeneous coordinate vectors. A scene point $X$ expressed in this frame projects in the $i^{t h}$ image onto the point $\mathrm{x}_{\mathrm{i}} \sim \mathrm{P}_{\mathrm{i}} \mathrm{X}(\sim$ is the equality up to a scale). We use $(.)_{\mathrm{k}}$ to refer to the $k^{t h}$ coordinate of the vector in argument and $(.)_{\mathrm{k} \ell}$ to refer to the entry at row $\mathrm{k}$ and column $\ell$ of the matrix in argument. The identity matrix and the null vector (dimensions are to be deduced from the context) are denoted by I and 0 .

Camera signatures and chirality: Camera (optical) centers $C_{i}$ are special points whose coordinates satisfy $\mathrm{P}_{\mathrm{i}} \mathrm{C}_{\mathrm{i}}=$ 0 . These coordinates can be obtained (with fixed scale) through the expansion of $\operatorname{det}\left(\begin{array}{c}\mathrm{P}_{i} \\ \Pi^{\top}\end{array}\right)=\Pi^{\top} C_{i}$ along the entries of the 4-vector $\Pi$. Scene points in front of a pair of cameras in the true metric configuration may appear in front of one camera but behind the other in a projective reconstruction. In such case, the cameras are said to be twisted by the projective transformation and untwisted otherwise. Based on Hartley's chirality theory [9] and using scene points that are visible by each camera pair, Nistér [12] proposed a simple yet robust twist test on cameras. The test is carried out by determining the signature $\zeta_{i} \in\{-1,+1\}$ 
of each camera as given by Algorithm 2, p.173 in [12]. Cameras with opposite signatures are twisted. A projective transformation that twists a pair of cameras moves the plane at infinity from its canonical position into one that cuts through the line segment joining their optical centers. All optical centers of cameras with positive signatures, i.e. $\zeta_{i}=1$, lie on one side with respect to the plane at infinity. Those with $\zeta_{i}=-1$ lie on the opposite side. The true plane at infinity, whose coordinate vector is denoted by $\Pi_{\infty}$, must satisfy

$$
\zeta_{i} \zeta_{j} \Pi_{\infty}^{\top} C_{i} C_{j}^{\top} \Pi_{\infty}>0 \text { for all } i, j=1, \ldots n .
$$

$\Pi_{\infty}$ being homogeneous, (1) can also be expressed by arbitrarily choosing a common sign $\zeta_{\mathrm{i}} \Pi_{\infty}^{\top} \mathrm{C}_{\mathrm{i}}>0($ or $<0)$ for all views. These inequalities are commonly known as the chirality inequalities of camera centers.

The Dual Absolute Quadric: The position of the plane at infinity is important in camera self-calibration as it allows to upgrade the projective scene and cameras to an affine frame. In particular, once its position is known, the interimage homographies induced by the plane at infinity can be recovered. Given the plane at infinity $\Pi_{\infty}^{\top}=\left(\pi_{\infty}^{\top} 1\right)$ and arbitrarily choosing the first camera as a reference, i.e. $P_{1}=[I \mid 0]$, such homographies relating the reference camera with each of the remaining cameras are given by

$$
\mathrm{P}_{\mathrm{i}}\left[\begin{array}{c}
\mathrm{I} \\
-\pi_{\infty}^{\top}
\end{array}\right]=\mu_{\mathrm{i}} \mathrm{KR}_{\mathrm{i}} \mathrm{K}^{-1} \text { for } i=2, \ldots n .
$$

In (2), each matrix $R_{i}$ is a $3 \times 3$ orthogonal matrix representing the rotation between the reference and the $i^{\text {th }}$ camera, $\mathrm{K}$ is a $3 \times 3$ upper-triangular matrix embedding the camera's intrinsic parameters, and $\mu_{i}$ scalars. Given(2), the following

$$
\mathrm{P}_{\mathrm{i}}\left[\begin{array}{cc}
\omega & \mathrm{n} \\
\mathrm{n}^{\top} & \mathrm{s}
\end{array}\right] \mathrm{P}_{\mathrm{i}}^{\top}=\lambda_{i} \omega, i=1 \ldots n
$$

holds for all views for some $\lambda_{i}>0$ (since $\lambda_{i}=\mu_{i}^{2}$ ). The $4 \times 4$ rank-3 symmetric positive semi-definite matrix $\left[\begin{array}{cc}\omega & n \\ n^{\top} & s\end{array}\right]$ represents the DAQ and the $3 \times 3$ symmetric positive-definite matrix $\omega=\mathrm{KK}^{\top}$ (with $\omega_{33}=1$ ) represents the DIAC, $\mathrm{n}=-\omega \pi_{\infty}$ and $\mathrm{s}$ a scalar. Finding the DAQ is equivalent to simultaneously locating the plane at infinity and the DIAC. Once the matrix $\omega$ is known, we extract the intrinsic parameters using Cholesky factorization.

Linear matrix inequalities: If matrix $A$ is positive-definite (resp. positive semi-definite), we denote $A>0$ or $-A<$ 0 (resp. $A \geq 0$ or $-A \leq 0$ ). Given matrices $A$ and $B$, $A>B$ (resp. $A \geq B$ ) means $A-B>0$ (resp. $A-$ $\mathrm{B} \geq 0$ ). A LMI is a constraint on a real-valued vector $\mathrm{y}=$ $\left(y_{0}, y_{1}, y_{2}, \ldots y_{m}\right)$ such that

$$
A(y)>0
$$

where $\mathrm{A}(\mathrm{y})=\mathrm{A}_{0}+\sum_{i=1}^{m} y_{i} \mathrm{~A}_{\mathrm{i}}$ is an affine function of $\mathrm{y}$ involving symmetric matrices $A_{0}, \ldots A_{m}$. A LMI may also be a negative definiteness constraint $A(y)<0$. While 4 is a strict LMI, the inequality therein may also be non-strict as to express positive (resp. negative) semi-definiteness $\mathrm{A}(\mathrm{y}) \geq 0$ (resp. $\mathrm{A}(\mathrm{y}) \leq 0$ ). A LMI feasibility problem is a convex optimization problem that can be solved very efficiently using interior-point methods [2]. Solving such problem means either finding y that satisfies the considered LMI or determining that no solution exists. From a practical point of view, some LMI softwares consider LMIs as nonstrict by default (SeDumi) while in others (Matlab Control Toolbox) inequalities are considered as strict. Note that, for a sufficiently small value $\epsilon>0$, a strict inequality (resp. non-strict), e.g. $A>0$ (resp. $A \geq 0$ ), can be turned into a non-strict $A \geq-\epsilon I$ (resp. strict $A>\epsilon I$ ) inequality. We recall that the largest eigenvalue $\lambda^{+}$and the smallest eigenvalue $\lambda^{-}$of a symmetric matrix $A$ satisfy $\lambda^{-} \mathrm{I} \leq \mathrm{A} \leq \lambda^{+} \mathrm{I}$. The GEVs of two matrices $A$ and $B$ are the values of $\lambda$ satisfying $\operatorname{det}(A-\lambda B)=0$. Given affine matrices $A(y)$ and $B(y)$ in $y$, the GEV problem consists in finding a y maximizing (resp. minimizing) their smallest (resp. largest) GEV. Maximizing the smallest GEV can be obtained by solving

$$
\begin{array}{ll}
\max _{\mathrm{y}} & \lambda \\
\text { s.t. } & \mathrm{A}(\mathrm{y}) \geq \lambda \mathrm{B}(\mathrm{y}), \mathrm{B}(\mathrm{y}) \geq 0, \mathrm{C}(\mathrm{y}) \geq 0
\end{array}
$$

possibly subject to additional constraints $\mathrm{C}(\mathrm{y})>0$. This is a quasiconvex problem that can be solved by bisection on $\lambda$. LMIs are used to solve a variety of problems which can be brought on the form (4) or its non-strict counterpart. In particular, some problems can be reformulated as LMIs by using the Schur complement lemma [2].

Lemma 2.1 Given a real symmetric block-partitioned matrix $\mathrm{D}=\left[\begin{array}{cc}\mathrm{A} & \mathrm{B} \\ \mathrm{B}^{\top} & \mathrm{C}\end{array}\right]$ and the Schur complement $\mathrm{S}=\mathrm{C}-\mathrm{B}^{\top} \mathrm{A}^{-1} \mathrm{~B}$ of (the symmetric block) $\mathrm{A}$ in $\mathrm{D}$,

$$
\text { if } \mathrm{A}>0 \text {, then } \mathrm{D} \geq 0 \Leftrightarrow \mathrm{S} \geq 0 \text {. }
$$

\section{LMI conditions on the DAQ}

Proposition 3.1 Let $\overline{\lambda_{i}^{-}}$be the maximum value of the smallest GEV obtained by solving

$$
\begin{array}{ll}
\max _{\omega, \mathrm{n}, s} & \lambda \\
\text { s.t. } & \mathrm{P}_{\mathrm{i}}\left[\begin{array}{cc}
\omega & \mathrm{n} \\
\mathrm{n}^{\top} & \mathrm{s}
\end{array}\right] \mathrm{P}_{\mathrm{i}}^{\top} \geq \lambda \omega, \Omega \geq 0, \quad \omega>0, \\
& \underline{(\omega)_{\mathrm{k} \ell}} \leq(\omega)_{\mathrm{k} \ell} \leq \overline{(\omega)_{\mathrm{k} \ell}}, k, \ell=1,2,3
\end{array}
$$

over the unknown vector $\mathrm{n}$, scalar $\mathrm{s}$ and the also unknown $\omega$ whose entries are bounded. If the entries of the true DIAC are within the bounds $(\omega)_{\mathrm{k} \ell}$ and $\overline{(\omega)_{\mathrm{k} \ell}}$, then $\overline{\lambda_{i}^{-}}$is bounded from above and the LMI

$$
\mathrm{P}_{\mathrm{i}}\left[\begin{array}{cc}
\omega & \mathrm{n} \\
\mathrm{n}^{\top} & \mathrm{s}
\end{array}\right] \mathrm{P}_{\mathrm{i}}^{\top} \leq \overline{\lambda_{i}^{-}} \omega
$$

is feasible for at least the true DIAC and DAQ. 
Proof The DAQ projection equation (3) conveys the information that, for the true DAQ and DIAC, the matrices $P_{i}\left[\begin{array}{cc}\omega & n \\ n^{\top} & s\end{array}\right] P_{i}^{\top}$ and $\omega$ admit $\lambda_{i}$ as a GEV since $\operatorname{det}\left(\mathrm{P}_{\mathrm{i}}\left[\begin{array}{cc}\omega & \mathrm{n} \\ n^{\top} & \mathrm{s}\end{array}\right] \mathrm{P}_{\mathrm{i}}^{\top}-\lambda_{i} \omega\right)=0$. More importantly, $\lambda_{i}$ is a GEV with algebraic multiplicity 3 . This can be easily seen by noticing that the GEVs of these two matrices are the eigenvalues of $\omega^{-1} \mathrm{P}_{\mathrm{i}}\left[\begin{array}{cc}\omega & n \\ n^{\top} & s\end{array}\right] \mathrm{P}_{i}^{\top}$ which simplifies to $\lambda_{i}$ I. As a consequence, if we allow $\omega$ and $\left[\begin{array}{cc}\omega & n \\ n^{\top} & s\end{array}\right]$ to possibly be the true DIAC and true DAQ, then $\overline{\lambda_{i}^{-}} \geq \lambda_{i}$ and the inequality (8) holds.

The claim in this proposition is that bounds on the entries of $\omega$ suffice for the GEV problem (7) to be bounded: no bounds on $\mathrm{n}$ and $\mathrm{s}$ are necessary. To demonstrate this, we use the fact that the DAQ projection equation (3) implies the well-known Kruppa's equations

$$
\left[q_{i}\right]_{\times} H_{i} \omega H_{i}^{\top}\left[q_{i}\right]_{\times}^{\top}=\lambda_{i}\left[q_{i}\right]_{\times} \omega\left[q_{i}\right]_{\times}^{\top}
$$

where $P_{i}=\left[H_{i} \mid q_{i}\right]$ and $\left[q_{i}\right]_{\times}$is the skew-symmetric matrix constructed from $q_{i}$ and associated with the crossproduct. Because of the congruence transformation any $\lambda$ satisfying $P_{i}\left[\begin{array}{ll}\omega & n \\ n^{\top} & s\end{array}\right] P_{i}^{\top} \geq \lambda \omega$ also satisfies $\left[q_{i}\right]_{\times} H_{i} \omega H_{i}^{\top}\left[q_{i}\right]_{\times}^{\top} \geq \lambda\left[q_{i}\right]_{\times} \omega\left[q_{i}\right]_{\times}^{\top}$. Hence the solution set of the former is included in the solution set of the latter. If the entries of $\omega$ are bounded, all entries of the matrices on both sides of the equality in (9) are also bounded. Moreover, because $\omega>0$, the diagonal entries of both matrices ought to be strictly positive. This implies that any $\lambda$ satisfying the LMI version of Kruppa's equation subject to $\omega>0$ must be bounded from above. Hence, the maximum of the smallest GEV of $P_{i}\left[\begin{array}{cc}\omega & n \\ n^{\top} & s\end{array}\right] P_{i}^{\top}$ and $\omega$ is also bounded.

This proof allows to deduce the following Corollary.

Corollary 3.2 Let $\overline{\lambda_{i}^{-}}$be the value of $\lambda$ obtained by solving the problem

$$
\begin{array}{ll}
\max _{\omega, \mathrm{n}, s} & \lambda \\
\text { s.t. } & {\left[\mathrm{q}_{\mathrm{i}}\right]_{\times} \mathrm{H}_{\mathrm{i}} \omega \mathrm{H}_{\mathrm{i}}^{\top}\left[\mathrm{q}_{\mathrm{i}}\right]_{\times}^{\top} \geq \lambda\left[\mathrm{q}_{\mathrm{i}}\right]_{\times} \omega\left[\mathrm{q}_{\mathrm{i}}\right]_{\times}^{\top},} \\
& \omega>0, \\
& \underline{(\omega)_{\mathrm{k} \ell}} \leq(\omega)_{\mathrm{k} \ell} \leq \overline{(\omega)_{\mathrm{k} \ell}}, k, \ell=1,2,3
\end{array}
$$

for some unknown but bounded $\omega$. If the entries of the true DIAC are within the bounds $(\omega)_{\mathrm{k} \ell}$ and $\overline{(\omega)_{\mathrm{k} \ell}}$, then $\overline{\lambda_{i}^{-}}$is bounded from above and the $\overline{L M I}(8)$ is feasible for at least the true DIAC and DAQ.

Proposition 3.3 Assuming $\omega>0, L M I$ (8) is feasible for a rank-3 matrix $\left[\begin{array}{cc}\omega & \mathrm{n} \\ \mathrm{n}^{\top} & \mathrm{s}\end{array}\right]$ where $\mathrm{s}=\mathrm{n}^{\top} \omega^{-1} \mathrm{n}$ if and only if (11) is feasible

$$
\left[\mathrm{P}_{\mathrm{i}}\left[\begin{array}{cc}
\omega \\
\omega \\
n^{\top}
\end{array}\right] \frac{[\omega n] P_{i}^{\top}}{\overline{\lambda_{i}^{-}} \omega}\right] \geq 0 .
$$

Proof The proof then boils down to applying Schur's lemma Lemma 2.1 after noticing that $P_{i}\left[\begin{array}{cc}\omega & n \\ n^{\top} & n^{\top} \omega^{-1} n\end{array}\right] P_{i}^{\top}=P_{i}\left[\begin{array}{c}\omega \\ n^{\top}\end{array}\right] \omega^{-1}\left[\begin{array}{ll}\omega & n\end{array}\right] P_{i}^{\top}$ and that $\overline{\lambda_{i}^{-}} \omega-\mathrm{P}_{\mathrm{i}}\left[\begin{array}{c}\omega \\ \mathrm{n}^{\top}\end{array}\right] \omega^{-1}[\omega \mathrm{n}] \mathrm{P}_{\mathrm{i}}^{\top}$ is the Schur complement of the matrix on the left hand-side of inequality 11 .

Proposition 3.4 The following LMI

$$
\zeta_{i} \zeta_{j} \mathrm{P}_{\mathrm{i}}\left[\begin{array}{cc}
\omega & \mathrm{n} \\
\mathrm{n}^{\top} & \mathrm{s}
\end{array}\right] \mathrm{P}_{\mathrm{j}}^{\top}+\zeta_{i} \zeta_{j} \mathrm{P}_{\mathrm{j}}\left[\begin{array}{cc}
\omega & \mathrm{n} \\
n^{\top} & \mathrm{s}
\end{array}\right] \mathrm{P}_{\mathrm{i}}^{\top} \geq 0
$$

is feasible for at least the true DAQ and DIAC when the rotation angle between two distinct views $i$ and $j$ of the camera (about an arbitrary axis) is at most $90^{\circ}$.

Proof The proof relies on the fact that $\mathrm{P}_{\mathrm{i}}\left[\begin{array}{cc}\omega & \mathrm{n} \\ n^{\top} & \mathrm{s}\end{array}\right] \mathrm{P}_{\mathrm{j}}^{\top}=\mu_{i} \mu_{j} \mathrm{KR}_{\mathrm{ij}} \mathrm{K}^{\top}$ (see 2p) where $R_{i j}=R_{i} R_{j}^{\top}$ is the rotation between the two views. Consider the eigendecomposition $\mathrm{R}_{\mathrm{ij}}=\mathrm{U} \operatorname{diag}\left(e^{J \theta}, e^{-J \theta}, 1\right) \mathrm{U}^{-1}$ where $\operatorname{diag}(\ldots)$ is the diagonal matrix of (unit norm, two of which are complex conjugate) eigenvalues of $R_{i j}$ and $\theta$ is the rotation angle between the two views. Because $\mathrm{R}_{\mathrm{ij}}^{-1}=\mathrm{R}_{\mathrm{ij}}^{\top}$, we also have $\mathrm{R}_{\mathrm{ij}}^{\top}=\mathrm{U} \operatorname{diag}\left(e^{-J \theta}, e^{J \theta}, 1\right) \mathrm{U}^{-1}$. We can hence deduce that the eigendecomposition of the symmetric matrix $R_{i j}+R_{i j}^{T}$ is of the form $\mathrm{R}_{\mathrm{ij}}+\mathrm{R}_{\mathrm{ij}}^{\top}=\mathrm{U} \operatorname{diag}(2 \cos (\theta), 2 \cos (\theta), 2) \mathrm{U}^{-1}$. For rotations not exceeding $90^{\circ}$, we have $R_{i j}+R_{i j}^{\top} \geq 0$ which remains true under a congruence transformation, i.e. $K\left(R_{i j}+R_{i j}^{\top}\right) K^{\top} \geq 0$. Since $\mathrm{P}_{\mathrm{i}}\left[\begin{array}{cc}\omega & \mathrm{n} \\ n^{\top} & \mathrm{s}\end{array}\right] \mathrm{P}_{\mathrm{j}}^{\top}+\mathrm{P}_{\mathrm{j}}\left[\begin{array}{cc}\omega & \mathrm{n} \\ n^{\top} & \mathrm{s}\end{array}\right] \mathrm{P}_{\mathrm{i}}^{\top}=\mu_{i} \mu_{j} \mathrm{~K}\left(\mathrm{R}_{\mathrm{ij}}+\mathrm{R}_{\mathrm{ij}}^{\top}\right) \mathrm{K}^{\top}$ matrix on the left-hand side is either positive or negative semi-definite. In particular, positive or negative definiteness is dependent upon the sign of $\mu_{i} \mu_{j}$ and can be set by considering the signatures of the involved projection matrices. Indeed, one my use (2) to show that $\mu_{i}^{3}=\operatorname{det}\left(\mathrm{P}_{\mathrm{i}}\left[\begin{array}{c}\mathrm{I} \\ -\pi_{\infty}^{\top}\end{array}\right]\right)=\operatorname{det}\left(\begin{array}{c}\mathrm{P}_{\mathrm{i}} \\ -\pi_{\infty}^{\top} 1\end{array}\right)$ which expands to $\mu_{i}^{3}=\Pi_{\infty}^{\top} \mathrm{C}_{\mathrm{i}}$. Because $\operatorname{sign}\left(\mu_{i} \mu_{j}\right)=\operatorname{sign}\left(\mu_{i}^{3} \mu_{j}^{3}\right)$, we have $\operatorname{sign}\left(\mu_{i} \mu_{j}\right)=\operatorname{sign}\left(\Pi_{\infty}^{\top} \mathrm{C}_{i} \mathrm{C}_{j}^{\top} \Pi_{\infty}\right)$. Using (1), we deduce that $\operatorname{sign}\left(\zeta_{i} \zeta_{j} \mu_{i} \mu_{j}\right)>0$ thus implying $\sqrt{12}$ is feasible.

\section{Proposition 3.5 If the LMIs}

$$
\begin{aligned}
& \omega>0 \\
& \zeta_{i} \zeta_{i+1} \mathrm{P}_{\mathrm{i}}\left[\begin{array}{cc}
\omega & \mathrm{n} \\
\mathrm{n}^{\top} & \mathrm{s}
\end{array}\right] \mathrm{P}_{\mathrm{i}+1}^{\top}+\zeta_{i} \zeta_{i+1} \mathrm{P}_{\mathrm{i}+1}\left[\begin{array}{cc}
\omega & \mathrm{n} \\
\mathrm{n}^{\top} & \mathrm{s}
\end{array}\right] \mathrm{P}_{\mathrm{i}}^{\top} \geq 0 \\
& \text { for all } \mathrm{i}=1 \ldots n
\end{aligned}
$$

are simultaneously feasible for some rank-3 $\left[\begin{array}{cc}\omega & n \\ n^{\top} & s\end{array}\right]$, then the candidate plane at infinity, with coordinates $\Pi=$ $\left(-\mathrm{n}^{\top} \omega^{-1} 1\right)^{\top}$, is guaranteed to satisfy chirality inequalities with respect to camera centers for all views; that is, all $\zeta_{i} \Pi^{\top} C_{i}$ for $\mathrm{i}=1 \ldots n$ carry the same sign. 
Proof The real parts of the eigenvalues of a matrix, whose sum with its transpose (i.e symmetric part) is definite, carry the same signs as those of its symmetric part. In our case, the eigenvalues of the matrix on the left-hand side of (13) are positive and so should be the determinant of $\zeta_{i} \zeta_{i+1} P_{i}\left[\begin{array}{cc}\omega & n \\ n^{\top} & s\end{array}\right] P_{i+1}^{\top}$. Furthermore, using the rank-3 condition on the quadric and $\omega>0$, imply that the feasible quadric must be of the form $\left[\begin{array}{c}I \\ n^{\top} \omega^{-1}\end{array}\right] \omega\left[I \omega^{-1} n\right]$. In this case, the determinant of $\zeta_{i} \zeta_{i+1} P_{i}\left[\begin{array}{cc}\omega & n \\ n^{\top} & s\end{array}\right] P_{i+1}^{\top}$ is positive if and only if the $\operatorname{det}\left(\zeta_{i}\left[\mathrm{I} \omega^{-1} \mathrm{n}\right] \mathrm{P}_{\mathrm{i}}^{\top}\right)$ and $\operatorname{det}\left(\zeta_{i+1}\left[\mathrm{I} \omega^{-1} \mathrm{n}\right] \mathrm{P}_{\mathrm{i}+1}^{\top}\right)$ carry the same sign. These determinants respectively expand to $\zeta_{i} \Pi^{\top} C_{i}$ and $\zeta_{i+1} \Pi^{\top} C_{i+1}$ hence demonstrating that both expressions carry the same sign which naturally generalizes to all camera centers when considering all views.

\section{LMI-based Branch-and-Prune DAQ search}

The B\&P algorithmic paradigm requires that an initial bounded space of the sought parameters, containing the optimal solution, to be given. The parameters' space is recursively subdivided into subspaces some of which are discarded while the others are kept for further investigation. This results in a dynamically generated search tree that is branched through subdivision and pruned through the elimination of subspaces. The B\&P algorithm we propose shares with the well-known $B \& B$ the branching and pruning of the parameters space. It however differs from $B \& B$ in that a bounding function on the targeted objective is not needed. Our method assumes that only bounds on the DIAC's parameters are available and no bounds on the plane at infinity are needed. Bounds on the intrinsic parameters can be easily translated into bounds on the 5 unknown entries of the DIAC's matrix $\omega$ while $(\omega)_{33}=1$. These bounds account for the initial parameters space to be subdivided and searched. At each iteration of our algorithm, we are given lower bounds $(\omega)_{k \ell}$ and upper bounds $\overline{(\omega)_{k \ell}}$ on the entries of $\omega$ for which a decision on whether this bounded subspace is to be investigated further or discarded following some LMI feasibility/infeasibility pruning conditions.

In the first step, one computes, for each view (except the first), the maximum value $\overline{\lambda_{i}^{-}}$of the smallest GEV by solving either problem (7) in Proposition 3.1 or (10) in Corollary 3.2 Note however that $[10]$ is computationally less expensive as it involves only 5 unknowns instead of 9 when using (7). We have used (10) in all the experiments whose results are reported in Section 5. GEV bounds obtained by (10) are generally less tight than those obtained by (7) possibly resulting in more branching. However, the subsequent pruning step, which we present next, is rather efficient in discarding parameter subspaces and the overall search is significantly faster when using $[10)$.
In the second step, we carry out a LMI feasibility/infeasibility pruning test within the bounds of $\omega$ at hand and involving simultaneously all views. The LMIs to be tested for feasibility/infeasibility depend on whether one would like to consider chirality of camera centers or not. We recall that the chirality/rotation LMIs (13) can be considered only when the rotation between consecutive images is known to be at most $90^{\circ}$. Given all $\overline{\lambda_{i}^{-}}$calculated in the previous step, the pruning test is as follows:

Rank-3 pruning test (without chirality): Test the simultaneous feasibility/infeasibility of LMIs (11) $i=2 \ldots n$ when considering all views with $\omega>0$ and the bound constraints $(\omega)_{\mathrm{k} \ell} \leq(\omega)_{\mathrm{k} \ell} \leq \overline{(\omega)_{\mathrm{k} \ell}}, \quad k, \ell=1,2,3\left(\omega_{33}=1\right)$. From Proposition 3.3. we understand that if these LMIs are infeasible, a positive semi-definite rank-3 quadric satisfying the DAQ projection equations (3) for all views is guaranteed not to exist for the specified bounds of $\omega$. In such case, the considered subspace defined by the bounds of $\omega$ is discarded. The subspace of $\omega$ is kept for further subdivision if these LMIs are simultaneously feasible.

Rank-3 and chirality test: Test the simultaneous feasibility/infeasibility of LMIs $(11) i=2 \ldots n$ along with the chirality/rotation LMIs (13) $i=1 \ldots n-1$ between consecutive views with $\omega>0,\left[\begin{array}{cc}\omega & n \\ n^{\top} & s\end{array}\right] \geq 0$ and the bound constraints $(\omega)_{\mathrm{k} \ell} \leq(\omega)_{\mathrm{k} \ell} \leq \overline{(\omega)_{\mathrm{k} \ell}}, k, \ell=1,2,3\left(\omega_{33}=1\right)$. If these LMIs are infeasible, then a positive semi-definite rank-3 quadric, satisfying (3) and leading to a candidate plane at infinity satisfying the chirality inequalities with respect to camera centers, is guaranteed not to exist within the considered bounds of $\omega$. This is due to Propositions 3.4 and 3.5. Again, the subspace of $\omega$ is kept for further subdivision if these LMIs are simultaneously feasible.

Every subspace of $\omega$ in which the LMIs are satisfied is considered "alive". Alive subspaces are stored in a list that is maintained sorted with respect to some objective evaluated at some feasible solution of the subspace. Although this might not be the best value of the objective in the considered subspace, we use this information as an indication on which subspace is more (or less) likely to contain the optimal solution. This indication becomes particularly more reliable with every reduction of the subspaces. The search tree generated by the subdivision of the space is explored following a best-first search strategy. This is carried out by visiting first, at each iteration, the alive subspace of parameters whose feasible solution yielded the smallest value of the objective. Branching is carried out along the longest edge; that is, assuming $\underline{(\omega)_{\mathrm{k} \ell}} \leq(\omega)_{\mathrm{k} \ell} \leq \overline{(\omega)_{\mathrm{k} \ell}}$, then the subspace is subdivided into two subspaces midway along the DIAC's parameter for which $\left|(\omega)_{k \ell}-\overline{(\omega)_{k \ell}}\right|$ is the largest. Each of these subspaces is then either alive (feasible LMIs) or discarded (infeasible LMIs). Note that a branch ceases to be explored when the bounds on $\omega$ are tight enough. The algo- 
rithm stops when the value of the objective for the best feasible solution is within some arbitrarily small value $\epsilon$ from the global optimum. If such solution fails to exist, the algorithm stops once there are no more alive subspaces and the best feasible solution is returned. All the results reported in this paper were obtained by minimizing (F: Frobenius)

$$
\max _{i=2 \ldots n}\left\|\frac{\mathrm{P}_{\mathrm{i}}\left[\begin{array}{cc}
\omega & \mathrm{n} \\
\mathrm{n}^{\top} & \mathrm{n}^{\top} \omega^{-1} \mathrm{n}
\end{array}\right] \mathrm{P}_{\mathrm{i}}^{\top}}{\left\|\mathrm{P}_{\mathrm{i}}\left[\begin{array}{cc}
\omega & \mathrm{n} \\
\mathrm{n}^{\top} & \mathrm{n}^{\top} \omega^{-1} \mathrm{n}
\end{array}\right] \mathrm{P}_{\mathrm{i}}^{\top}\right\|_{F}}-\frac{\omega}{\|\omega\|_{F}}\right\|_{F} .
$$

Remark: Note that, as in [7], relaxing the rank constraint (for a posteriori enforcement) is possible in our method and boils down to checking the infeasibility of $(8)$ instead of that of (11) after solving the GEV problems. However, while enforcing the rank a posteriori works well in [7] for the Dual Linear method, it proved computationally inefficient for our B\&P algorithm. This is because subspaces containing only full-rank quadrics satisfying (8) are not pruned but rather kept for further investigation yielding an increase in the branching and hence more GEVs and LMIs to solve.

\section{Experiments}

We tested our method using synthetic and real images. Normalization of image points was used throughout. Projective reconstruction was obtained by [8] and refined via Bundle Adjustment (BA) using Rabaud's SfM Toolbox vision. ucsd.edu/ vrabaud. The algorithm was implemented in MATLAB2012a and all the LMI problems were solved using its LMI control toolbox. All experiments were carried out on a Pentium i5/2.50GHz/6GB RAM.

Simulations: We generated a set of 120 random 3D points scattered within a sphere of radius 100 units for each scene simulation. The cameras were placed about $280 \pm 75$ units away from the center of the sphere. The motion of the camera was restricted so that all views face the scene and the rotation between consecutive images was at most $90^{\circ}$ about an arbitrary axis passing nearby the center of the sphere. All scene points were projected on $256 \times 256$ images using a camera of constant intrinsic parameters with horizontal and vertical focal lengths $f_{x}=f_{y}=300$, zero-skew $\alpha=0$, and principal point $u_{0}=128$ and $v_{0}=128$. The synthetic images were obtained by adding various levels of zero-mean Gaussian noise to the pixel coordinates. For each image sequence length and noise level (std. dev. from 0 to 2.0 with 0.5 step), we ran 100 independent tests. We conducted two separate tests with and without our chirality/rotation LMIs. For each data set, the stratified method [3], starting form exactly the same initial bounds on the intrinsic parameters, was also used for comparison. The results obtained were refined by minimizing the sum of squares of the DAQ projection error using Levenberg-Marquardt algorithm. A test was considered successful if the individual error in focal lengths and principal points are within $20 \%$ and $|\alpha|<20$. The accuracy of the $3 \mathrm{D}$ reconstruction was evaluated by computing the RMS error after aligning the reconstructed point cloud with the original cloud through the best metric transformation in a least-square sense. Both point clouds are normalized while keeping their mean distance equal to $\sqrt{2}$ before computing the error. The projective structure is upgraded to metric with the help of the change of basis transformation, which can easily be improved by other refinement techniques like BA. If $f_{x}^{i}, f_{y}^{i}, \alpha^{i}, u_{0}^{i}, v_{0}^{i}, i=$ $1 \ldots N$ are intrinsic parameters obtained form $N$ different calibrations, the RMS errors are computed as follows: $\Delta f_{r m s}=\left(\frac{\sum_{i=1}^{N}\left(f_{x}^{i}-f_{x}\right)^{2}+\left(f_{y}^{i}-f_{y}\right)^{2}}{N\left(\left(f_{x}\right)^{2}+\left(f_{y}\right)^{2}\right)}\right)^{1 / 2}$, $\Delta u v_{r m s}=\left(\frac{\sum_{i=1}^{N}\left(u_{0}^{i}-u_{0}\right)^{2}+\left(v_{0}^{i}-v_{0}\right)^{2}}{N\left(\left(u_{0}\right)^{2}+\left(v_{0}\right)^{2}\right)}\right)^{1 / 2}$ and $\Delta \alpha_{r m s}=\left(\frac{\sum_{i=1}^{N}\left(\alpha^{i}-\alpha\right)^{2}}{N}\right)^{1 / 2}$. Various measurements of the success rate and accuracy for different methods are shown in Figures 14 Our results suggest that the proposed method is stable from as few as 4 views. When rotation LMIs are added or more views are used, the success rate improves as expected. Our method does not consider all the reconstructed points to be in front of the camera, which in fact may be unreliable in the presence of noise. This may be one reason behind the failure of the stratified method. On the other hand, two globally optimal solutions of the stratified method may not necessarily be the global solution as a whole. It is important to notice that there may be multiple global solutions in some cases. We have however considered only the first solution found. The termination of the algorithms before exploring all possible solutions could be another reason of failure in such cases for both methods. Incorporating the chirality/rotation LMIs and/or increasing the number of views customarily decreases the $3 \mathrm{D}$ reconstruction error. The results in Figure 2(right) show few abnormalities with higher levels of noise in 5-7 views. This is probably due to the change in the number of samples for error computation. The samples that would have failed with only 5 views but succeeded with 7 are likely to give larger errors and hence increase the overall error. Our method with and without the rotation LMIs, as shown in Figure 3 is significantly faster than the stratified one while providing good quality intrinsic parameters (Figure 4 for 5 views) that require only little refinement. It is observed that the optimization with chirality/rotation LMIs is computationally more expensive. This results in an increase of the overall running-time even when the number of iterations is substantially decreased. Note that we have been unable to conduct a meaningful comparison of our method against the polynomial method in [4]. Unlike in [4], in most cases with our data, the method failed to converge to the optimal DAQ with a relaxation order of 2 . We found that [4] gives the 

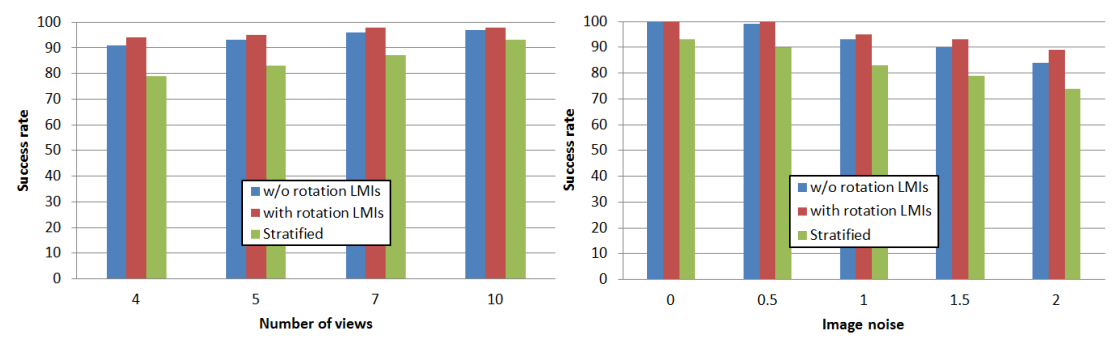

Figure 1. Success rate for 1 pixel noise (left) and 5 views (right).

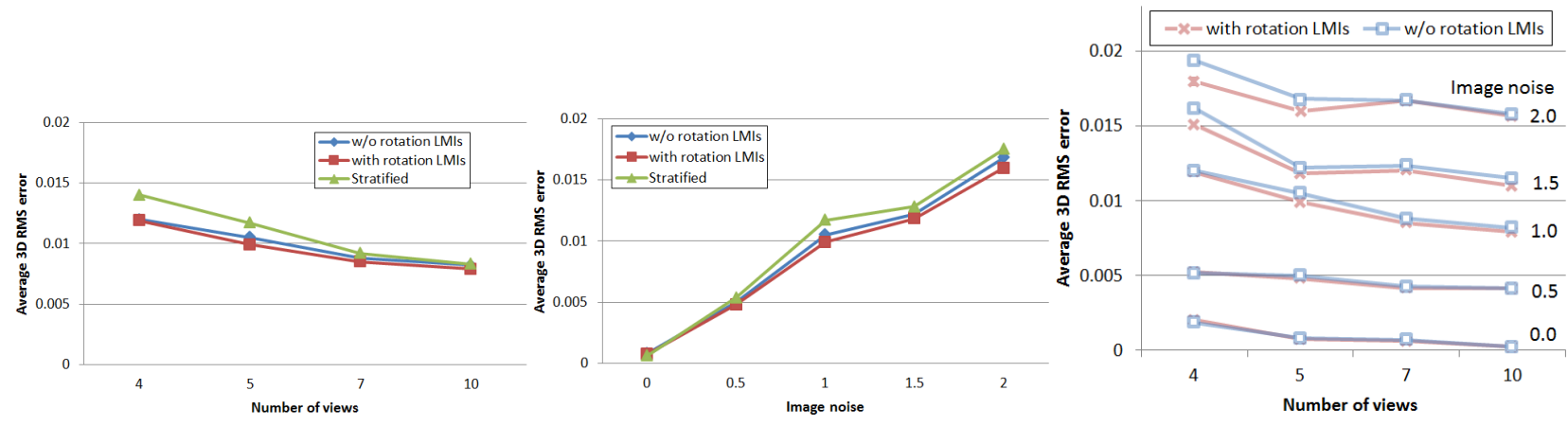

Figure 2. 3D RMS error for 1 pixel noise (left), 5 views (middle), and variable views and noise (right).

right results with an order of relaxation 3 but with about 30 minutes of running-time on the conducted tests.

Real Images: We present the results obtained with three image sequences. Our method without chirality/rotation LMIs, with chirality/rotation LMIs and the stratified method are labeled as A, B and C respectively. The first sequence consists of $93000 \times 4000$ images of a standard-sized football, Figure 5(left), in which 14 common points were manually selected. These points are corners of the truncated icosahedron present on its surface. The wire-frame reconstruction of our method with chirality/rotation LMIs is shown in Figure 5 (middle-right). In this figure, the structure was upgraded to Euclidean (the real world measurement of the sides of polygons is known to be $4.5 \mathrm{~cm}$ ) and a sphere was fitted to the reconstructed 3D points and shown. The qualitative results of our method with and without chirality/rotation LMIs are not shown separately because of them being very similar. However, the quantitative results are presented. The following geometric parameters were computed and compared in Table 1 against those of FIFA:

1. LS: RMS error of the length of sides.

2. AH: RMS error of the internal angles of hexagons.

3. AP: RMS error of the internal angles of pentagons.

4. A-HP: Mean(M) and Variance(V) of Dihedral angles between hexagons and pentagons (expected: 142.62).

5. A-HH: Dihedral angle between two hexagons (expected: 138.19).

6. CS: Sphere circumference (expected: 68-70 cm).
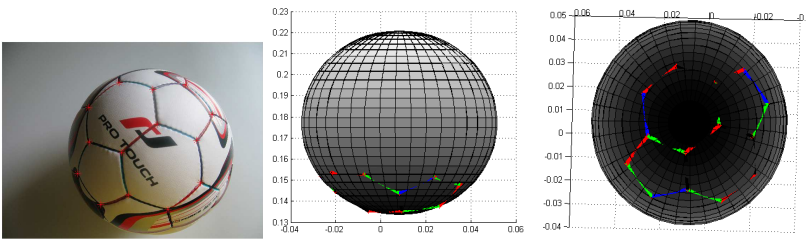

Figure 5. Football (left), fitted sphere/Method B (middle-right)

\begin{tabular}{|c|c|c|c|c|c|c|c|}
\hline & \multirow{2}{*}{$\begin{array}{c}\mathrm{LS} \\
(\mathrm{cm})\end{array}$} & \multirow{2}{*}{$\mathrm{AH}$} & \multirow{2}{*}{ AP } & \multicolumn{2}{|c|}{ A-HP } & \multirow{2}{*}{ A-HH } & \multirow{2}{*}{$\begin{array}{c}\mathrm{CS} \\
(\mathrm{cm})\end{array}$} \\
\hline & & & & $\mathrm{M}$ & V & & \\
\hline $\mathrm{A}$ & 0.16 & 1.01 & 0.88 & 141.52 & 2.53 & 135.93 & 68.05 \\
\hline B & 0.16 & 1.03 & 0.083 & 141.51 & 2.54 & 135.93 & 68.06 \\
\hline $\mathrm{C}$ & 2.12 & 5.64 & 11.03 & 161.37 & 1.57 & 160.48 & 134.63 \\
\hline
\end{tabular}

Table 1. Geometric measurements.

The second image sequence we used is the "Model house" from www.robots.ox.ac.uk/ vgg with $95 \mathrm{im}$ age points common in $6576 \times 768$ images. Our methods with and without chirality/rotation LMIs produced the same results whereas the stratified method failed to provide a valid result. Figure 6 shows one of the images and two views of the reconstructed surface before and after texture mapping. The ground truth being available, the 3D RMS error computed for this sequence is 0.0109 .

The third image sequence (results in Table 2 ) consisted of $81200 \times 1600$ images (not shown) with 16 feature points common to all views. The camera parameters were obtained using the pattern-based calibration technique followed by methods A, B and C. 

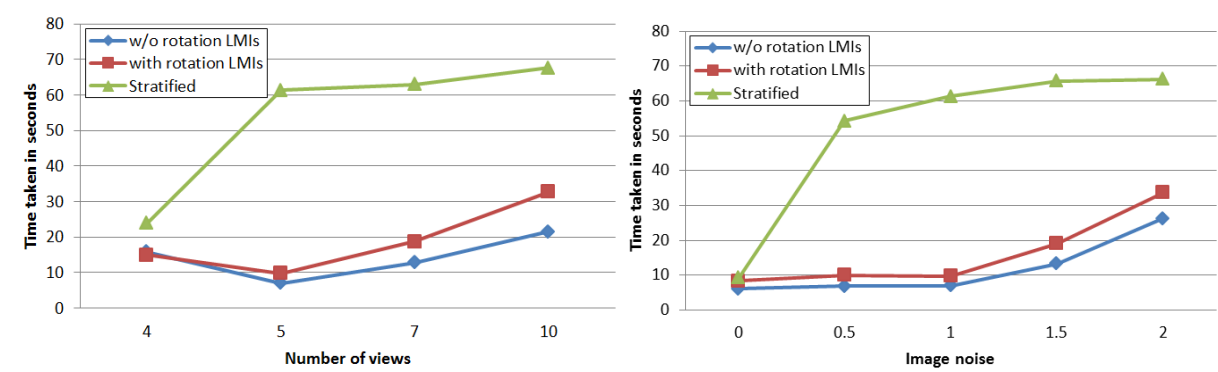

Figure 3. Median time taken for 1 pixel noise (left) and 5 views (right).
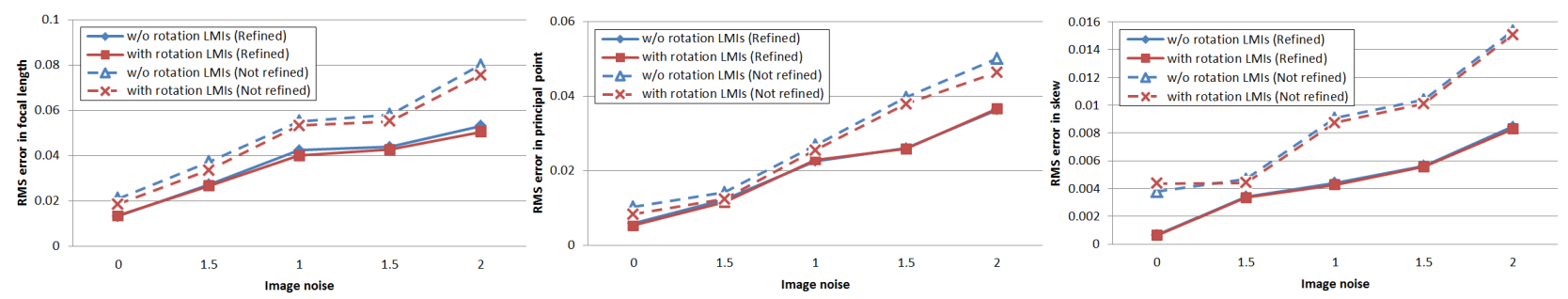

Figure 4. RMS error in intrinsic parameters before and after the refinement for 5 views.
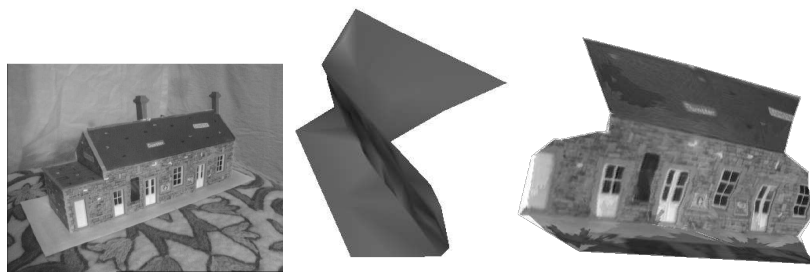

Figure 6. Model house reconstruction with method B.

\begin{tabular}{|c|c|c|c|c|c|}
\cline { 2 - 6 } \multicolumn{1}{c|}{} & $f_{x}$ & $f_{y}$ & $u_{0}$ & $v_{0}$ & $\alpha$ \\
\hline $\mathrm{A}$ & 1615.49 & 1610.70 & 810.73 & 615.21 & 0.63 \\
\hline $\mathrm{B}$ & 1611.90 & 1610.45 & 812.09 & 611.55 & 0.49 \\
\hline $\mathrm{C}$ & 1603.86 & 1597.58 & 749.04 & 645.37 & -32.29 \\
\hline Pattern-based & 1601.32 & 1606.14 & 808.87 & 613.59 & 0.00 \\
\hline
\end{tabular}

Table 2. Camera intrinsic parameters.

\section{Conclusion}

We have presented a new globally optimal algorithm for estimating the DAQ under the rank-3 and, optionally, camera centers chirality constraints. The proposed method employs the Branch-and-Prune algorithmic paradigm and requires to explore the space of only 5 parameters. Our algorithm relies on some new results providing LMI feasibility/infeasibility pruning conditions. Our LMI chirality/rotation conditions were obtained under the mild assumption that the camera undergoes a rotation of no more than $90^{\circ}$ between consecutive views.

\section{References}

[1] Bocquillon B., Bartoli A., Gurdjos P., Crouzil A.: On Constant Focal Length Self-Calibration From Multiple Views. CVPR. (2007) 1-8
[2] Boyd, S., Vandenberghe, L.: Convex Optimization. Cambridge University Press (2004)

[3] Chandraker, M., Agarwal, S., Kriegman, D., Belongie, S.: Globally Optimal Algorithms for Stratified Autocalibration. IJCV 90 (2010) 236-254

[4] Chandraker, M., Agarwal, S., Kahl, F., Kriegman, D., Nister, D.: Autocalibration via Rank-Constrained Estimation of the Absolute Quadric. CVPR (2007) 1-8

[5] Fusiello, A., Benedetti, A., Farenzena, M., Busti, A. Globally convergent auto-calibration using interval analysis. IEEE Trans. PAMI 26 (2004) 1633-1638

[6] Gherardi, R., Fusiello, A.: Practical Autocalibration. ECCV 2010, Part I. Vol. 6311 of LNCS, Springer (2010) 790-801

[7] Gurdjos, P., Bartoli, A., Sturm, P.F.: Is Dual Linear Self-calibration Artificially Ambiguous? ICCV. (2009) 88-95

[8] Oliensis I., Hartley R.: Iterative extensions of the sturm/triggs algorithm: Convergence and nonconvergence. IEEE Trans. PAMI, 29(12):2217-2233, 2007.

[9] Hartley, R.I., Zisserman, A.: Multiple View Geometry in Computer Vision. Second edn. Cambridge University Press (2004)

[10] Heyden, A., Åström, K.: Euclidean Reconstruction from Constant Intrinsic Parameters. CVPR. Vol. 1., (1996) 339-343

[11] Luong, Q.: Self-calibration of a Moving Camera from Point Correspondences and Fundamental Matrices. IJCV 22 (1997) 261-289

[12] Nistér, D.: Untwisting a Projective Reconstruction. IJCV 60 (2004) $165-183$

[13] Pollefeys, M., van Gool, L.: Stratified Self-calibration with the Modulus Constraint. IEEE Trans. PAMI 21 (1999) 707-724

[14] Pollefeys, M., Koch, R., Gool, L.V.: Self-calibration and Metric Reconstruction in spite of Varying and Unknown Internal Camera Parameters. IJCV 32 (1999) 7-25

[15] Triggs, B.: Autocalibration and the Absolute Quadric. CVPR (1997) 609-614 